\title{
Mother-to-infant transmission of the carcinogenic colibactin-producing bacteria
}

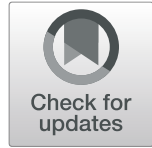

\author{
Yuta Tsunematsu', Koji Hosomi ${ }^{2}$, Jun Kunisawa ${ }^{2}$, Michio Sato ${ }^{1}$, Noriko Shibuya ${ }^{3}$, Emiko Saito ${ }^{4}$, Haruka Murakami ${ }^{5}$, \\ Yuko Yoshikawa ${ }^{6}$, Yuji Iwashita', Noriyuki Miyoshi ${ }^{7}$, Michihiro Mutoh ${ }^{9}$, Hideki Ishikawa ${ }^{9}$, Haruhiko Sugimura ${ }^{7}$ \\ Motohiko Miyachi ${ }^{5}$, Keiji Wakabayashi ${ }^{8}$ and Kenji Watanabe ${ }^{1^{*}}$ (i)
}

\begin{abstract}
Background: The Escherichia coli strain that is known to produce the genotoxic secondary metabolite colibactin is linked to colorectal oncogenesis. Therefore, understanding the properties of such colibactin-positive $E$. coli and the molecular mechanism of oncogenesis by colibactin may provide us with opportunities for early diagnosis or prevention of colorectal oncogenesis. While there have been major advances in the characterization of colibactinpositive $E$. coli and the toxin it produces, the infection route of the $c l b+$ strain remains poorly characterized.

Results: We examined infants and their treatments during and post-birth periods to examine potential transmission of colibactin-positive E. coli to infants. Here, analysis of fecal samples of infants over the first month of birth for the presence of a colibactin biosynthetic gene revealed that the bacterium may be transmitted from mother to infant through intimate contacts, such as natural childbirth and breastfeeding, but not through food intake.

Conclusions: Our finding suggests that transmission of colibactin-positive E. coli appears to be occurring at the very early stage of life of the newborn and hints at the possibility of developing early preventive measures against colorectal cancer.
\end{abstract}

Keywords: Colibactin, Natural product, Escherichia coli, Perinatal transmission, Colorectal cancer

\section{Background}

Colorectal cancer (CRC) is the third most common form of cancer and the second most common cause of cancer mortality in the world [1]. It is predicted that by 2030 approximately 2.2 and 1.1 million people will develop CRC and succumb to it [2]. To reduce the number of CRC incidences and mortalities, it is vital to identify and mitigate the source of risk factors that contribute to the onset of CRC. Certain strains of Escherichia coli that harbor the gene cluster $c l b$ (also referred to as $p k s$ ) responsible for the biosynthesis of the genotoxin colibactin have been linked to colorectal oncogenesis [3-10].

\footnotetext{
*Correspondence: kenji55@u-shizuoka-ken.ac.jp

'Department of Pharmaceutical Sciences, University of Shizuoka, 422-8526 Shizuoka, Japan

Full list of author information is available at the end of the article
}

Recently, we created fluorescent probes [11] that are turned on specifically by ClbP, a peptidase required to activate the prodrug-like colibactin precursor [12, 13]. The probe allowed high-throughput screening of E. coli isolates from clinical samples, which led to the isolation of the high-colibactin producer $E$. coli-50 that will be useful in studying the properties of colibactin-positive $(\mathrm{clb}+)$ E. coli and colibactin [11]. While we continue to elucidate the molecular mechanism of oncogenesis by $c l b+E$. coli and colibactin, there is still a limited understanding on the infection route of the E. coli to the affected individuals. Since identification of the routes of infection would help develop measures to mitigate or prevent the infection, we initiated a screening effort to examine the prevalence of $c l b+E$. coli among healthy individuals by analyzing their fecal samples [14] and are 
currently investigating the continued $c l b+E$. coli infections that occur among healthy individuals. Also, it is known that the newborn gut microbiota starts to form upon exposure to the vaginal and maternal skin microbiomes after birth [15]. A study based on a rat model showed that commensal E. coli strains, including $\mathrm{clb}+E$. coli, are transmitted from mothers to neonates, where early colonization of neonate gut with genotoxic $E$. coli could influence the intestinal homeostasis at adulthood in a way that may put the individual at risk of colorectal cancer and other immune-mediated diseases [16]. Therefore, we extended our screening to include newborns to study our main clinical objective, which is to determine if $\mathrm{clb}+$ E. coli could be transmitted to infants from mother or closely interacting caretaking adults, and if so, what is the source and the medium through which the strain is being passed on to the infants. Here we report that the colibactin-producing $E$. coli can indeed be rapidly transmitted from mother to child after birth, suggesting that a respectable number of healthy individuals may become predisposed to high risk of CRC at the very early stage of life.

\section{Results}

In a previous study we conducted to identify the frequency of healthy adults who carry $c l b+E$. coli, we surveyed 223 healthy adults from Tokyo metropolitan area in Japan for the presence of $c l b+E$. coli in their fecal samples. We found that 60 participants $(26.9 \%)$ were positive [14]. We extended the study further to investigate the timing at which individuals become infected with $c l b+E$. coli. For the current study, we examined 51 infants ( 25 male, 26 female). From the subjects, one set of feces was collected at birth or within two to three days after birth, and another set was collected one month after birth. The samples were examined by PCR to detect the presence of $c l b B$, the gene for one of the PKS-NRPS hybrid megasynthetases encoded in the colibactin biosynthetic gene cluster. We found that 8 out of 51 newborns or $15.7 \%$ of the test samples harbored $c l b+$ E. coli immediately after birth (Fig. 1a, lanes 7, 10, 17, 22, 25, 34, 45 and 47 and Fig. 2). On the other hand, 16 of the $51(31.4 \%)$ tested positive for $c l b+E$. coli one month after birth (Fig. 1b, lanes 7, 10, 17, 18, 22, 25, 26, 27, 28, 29, 31, 34, 35, 45, 47 and 49 and Fig. 2), indicating that 8 newborns or $15.7 \%$ acquired the $c l b+E$. coli strain during their first month. The $c l b$-positive rate increased from 15.7 to $31.4 \%$ by the end of the first month, reaching to the equivalent level of $26.9 \%$ observed among healthy adults examined recently [14].

Perinatal transmission can happen in utero, in the birth canal or through breastfeeding. Because intrauterine transmission of $E$. coli in healthy pregnancies is considered to be infrequent [17], we next investigated the correlation between the method of delivery and the clbpositive ratio among the newborns we studied. Because we expected that the delivery method would only affect the $c l b$-positive ratio among newborns to a few-days-old babies, we did not employ the data collected one month after birth. Regarding the birth canal transmission, $c l b+$ E. coli was detected in seven out of eight or $87.5 \%$ of the infants that were born through natural delivery (Table 1). In contrast, only one of eight or $12.5 \%$ of the infants that were delivered by Cesarean section was $c l b$ positive (Table 1).

As to the breastfeeding-mediated transmission, we examined the correlation between the infant feeding mode and the $c l b$-positive ratio. We screened the 43 infants who were determined to be $c l b$-negative at birth to a few days after birth. After one month, eight of the $43 \mathrm{clb}$ negative infants presented $c l b$-positive, while the remaining $35(81.4 \%)$ were not affected as determined by the PCR analysis of their fecal samples (Table 2). In total, there were 26 and 17 infants who were fed breastmilk alone and a mixture of formula and breastmilk, respectively. Among the eight $c l b$-positive infants, seven were breastfed strictly over the one-month period, whereas only one newborn was fed a mixture of formula and breastmilk over the month. While $26.9 \%$ of those given breastmilk alone became $c l b$-positive, only $5.9 \%$ of the infants given a mixed feed became $c l b$-positive (Table 2). To check if intaking or handling of food items by caretakers could play a role in transmitting $c l b+E$. coli to the infants, 58 different samples collected from food items including tap water that are commonly consumed by the demographics examined in the current study were screened for the presence of $c l b+E$. coli (Table 3). The search failed to identify $c l b+E$. coli except in a sample of cattle stomach. However, the strain found in the sample of cattle stomach belonged to the phylogroup B1, whereas all of the $c l b+E$. coli strains isolated from human subjects thus far belonged to B2 (manuscript in preparation, Y.Y., Y.T., M.S., Y.I., N.M., M.Mutoh., H.I., H.S., K.Wakabayashi and K.Watanabe). Lastly, we did not observe any difference in the $c l b+E$. coli infection ratio between the sexes of the infants either at birth or one month after birth in this study.

\section{Discussion}

Our survey of infants identified that only $15.5 \%$ of the screened infants harbored $c l b+E$. coli immediately after birth (Fig. 1a, lanes 7, 10, 17, 22, 25, 34, 45 and 47 and Fig. 2). However, after one month the percentage doubled to $31.4 \%$, similar to the frequency found among healthy adults [14] (Fig. 1b, lanes 7, 10, 17, 18, 22, 25, 26, 27, 28, 29, 31, 34, 35, 45, 47 and 49 and Fig. 2). Once infected, it is expected that $c l b+E$. coli remains within the system of the infected individual persistently. Thus, 


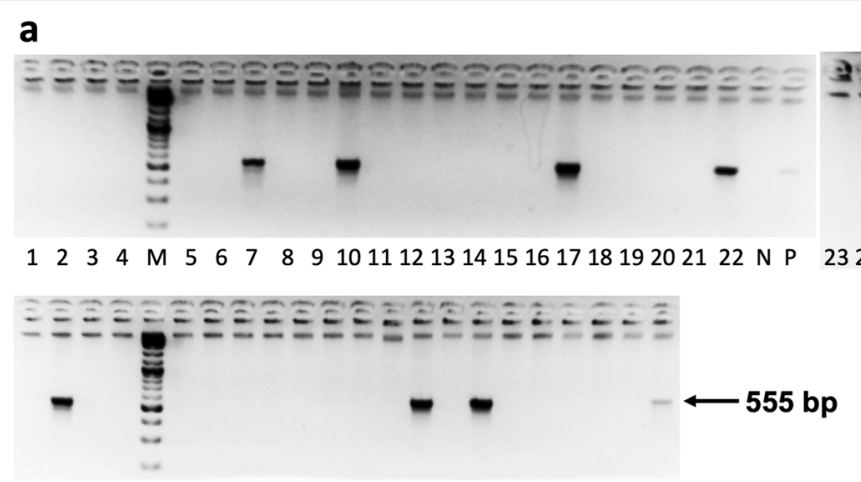

33343536 M 373839404142434445464748495051 N P

b
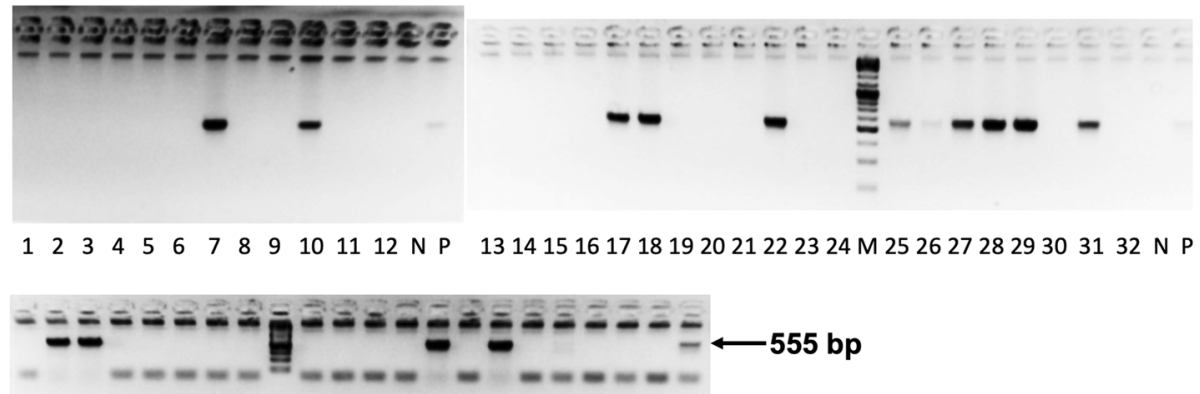

3334353637383940 M $4142434445464748495051 \mathrm{~N} \quad \mathrm{P}$

Fig. 1 PCR analysis for the presence of $c l b+E$. coli in fecal samples of infants. Amplifications of PCR products from the DNA samples extracted from fecal samples was performed using the primer set described in Online Methods. (a) Results from the fecal samples from infants collected at birth to two to three days after birth. (b) Results from the fecal samples collected one month after birth. Lane M: molecular weight marker; lane $\mathrm{N}$ : negative control using pUC19 as a PCR template; lane P: positive control using the gDNA isolated from E. coli-50[1 1] as a PCR template; lanes 1-51: analysis of the sample from each of the 51 newborn subjects. The size of the expected $\mathrm{llbB}$ amplicon generated with the primer set $\mathrm{clb}-\mathrm{F} /$ clb-R is 555 bp. Full-length gels are presented in Supplementary Fig. 1 of the Supplementary Information

the adult-like $c l b$-positive rate we found among the onemonth-old newborns in this study suggests that healthy individuals become infected with $\mathrm{clb}+E$. coli very early in their life stage in Japan. These results also indicate that the infants are getting exposed to the source of $c l b+$ E. coli under the ordinary living condition during its first month of life.

Next, we examined the source of perinatal transmission, where we focused on the correlation between the method of delivery and the $c l b$-positive ratio among the newborns. The study found that while $87.5 \%$ of the infants delivered by natural birth were $c l b$-positive, only $12.5 \%$ of those delivered by Cesarean section were $c l b$ positive (Table 1). Those results indicated that a higher $c l b$-positive ratio was observed among infants that were born through natural childbirth, similar to how the chance of infants acquiring the vaginal flora bacteria, including $E$. coli, increases by passing through the birth canal.

We also examined the possible role breastfeeding plays in the transmission of $c l b$-positive $E$. coli to infants. Of the $c l b$-negative infants that were strictly breastfed, $26.9 \%$ became $c l b$-positive one month after birth, while only $5.9 \%$ of those given a mixture of formula and breastmilk turned $c l b$-positive (Table 2). A simple survey of food items that are considered to be consumed typically by the demographics to which infant caretakers belong showed that none of the food samples examined was contaminated by the $c l b+E$. coli strains that are isolated from human subjects. Therefore, intaking or handling of food items by caretakers being a potential source of $c l b+E$. coli being transmitted to infants was considered less likely. The results implicate that the transmission of clb-positive $E$. coli to infants occurs mainly through mothers having close contacts with the infants.

\section{Conclusions}

In summary, the infection ratio of $c l b+E$. coli is $31.4 \%$ among the one-month-old infants studied, which is similar to the frequency found among healthy adults [14]. It was also reported that 130 Swedish infants followed from birth to 18 months of age were determined to be $33 \% \mathrm{clb}$ - 


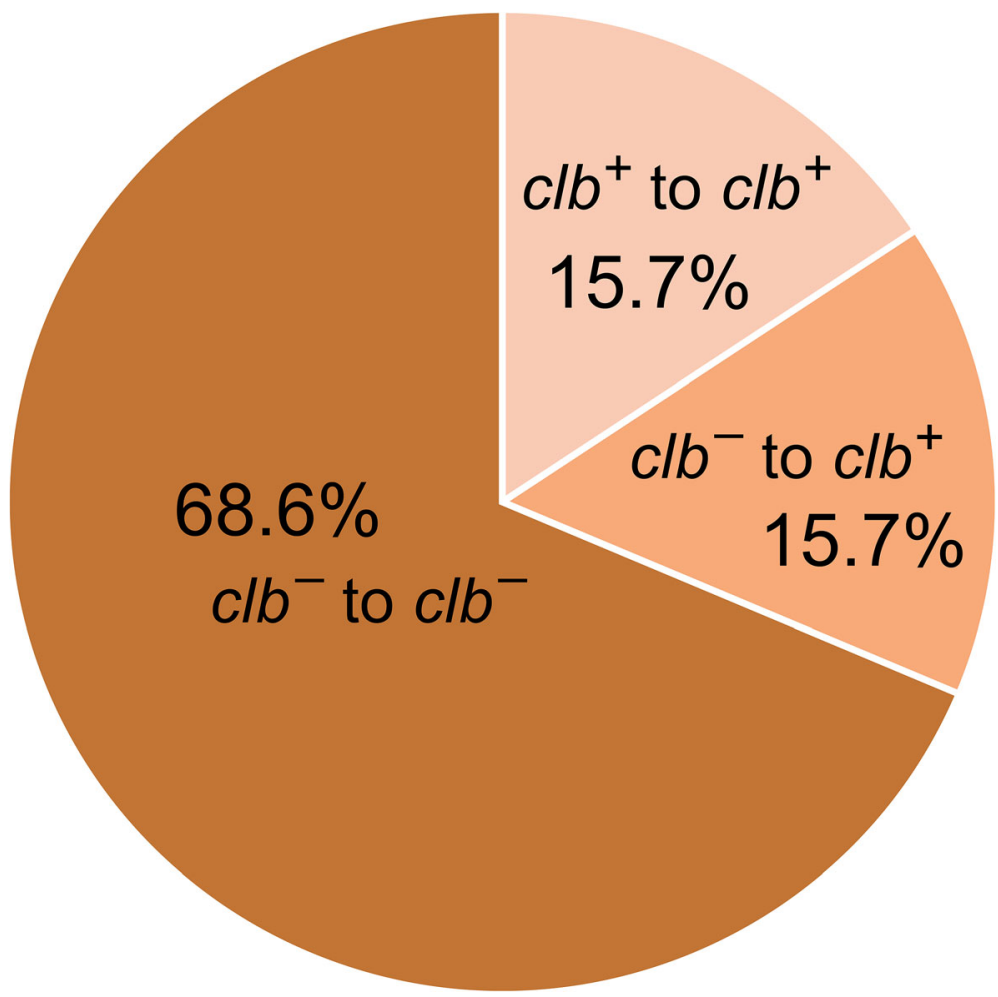

Fig. 2 The frequency of diagnosis for $\mathrm{clb}+$ and $\mathrm{clb}$ - among the newborns. The $\mathrm{clb}$-positive rate among the newborns at birth to a few days after birth is $15.7 \%$, and one month that is $15.7 \%(\mathrm{clb}+$ to $\mathrm{clb}+)$ plus $15.7 \%(\mathrm{clb}-$ to $\mathrm{clb}+), 31.4 \%$ in total. Infants were screened within two to three days of birth after one month of either mixed feeding or breastfeeding alone

positive, very similar to our findings of $31.4 \%$, when their feces were analyzed using a similar PCR method [18]. Our analysis identified that infants born by natural delivery had a higher incidence of being $c l b$-positive than those born by Cesarean section. Similarly, those who were breastfed strictly showed a higher clb-positive frequency than those given a mixed feed. The fact that the mixed feed also contained breastmilk suggests that breastmilk itself was not the source of $c l b+E$. coli. A simple survey of food items commonly consumed by the Japanese also indicated that $c l b+E$. coli was probably not transmitted through contaminated food consumed or handled by caretakers of the infants. Rather, the most probable route of infection of the potentially oncogenic $c l b+E$. coli strain appears to be through direct skin-to-skin contact, or skinto-mouth contact involved in breastfeeding to be more specific, between mother and her infant (Table 2). The same can be said about the method of delivery, where infants born by natural birth would have a substantially higher frequency of direct skin-to-skin contact with its mother than those born by Cesarean section (Table 1). Taken together, similar to how the gut microbiota is transmitted from mother to infant [19], our results strongly imply that $c l b+E$. coli might be transmitted from mother to newborn from the very early stage of life of the newborn through intimate contacts with the mother. Therefore, by implementing measures that can reduce the transmission of $c l b+E$. coli from adults to infants, we may be able to lower the incidence of CRC in our population. For instance, we would be able to develop early preventive measures against colorectal cancer. Those measures could include procedures to prevent infection during childbirth,

Table 1 The correlation between the frequency of infection with $c l b+E$. coli among 51 infants examined and their delivery methods. Number of infants $(n=51)$

\begin{tabular}{|c|c|c|c|c|c|}
\hline \multirow[b]{2}{*}{ Delivery method } & \multicolumn{2}{|c|}{ No. of infants $(n=51)$} & \multirow[b]{2}{*}{ Total } & \multirow[b]{2}{*}{ OR (95\% Cl) } & \multirow[b]{2}{*}{$p$-value } \\
\hline & $c l b+$ & $c l b-$ & & & \\
\hline Natural delivery & 7 & 35 & 42 & $1.6(0.17$ to 14.90$)$ & 0.67 \\
\hline Cesarean section & 1 & 8 & 9 & 1 & \\
\hline
\end{tabular}


Table 2 The correlation among the frequency of diagnosis for $\mathrm{clb}+\mathrm{vs}$. $\mathrm{clb}$ - and provision of one month of mixed feeding vs. breastfeeding alone among the newborns who tested negative at birth or two to three days after birth. For each category, breakdown of the cases based on delivery methods is also given. Number of infants $(n=43)$

\begin{tabular}{|c|c|c|c|c|c|}
\hline \multirow[b]{2}{*}{ Feeding } & \multicolumn{2}{|c|}{ No. of infants $(n=51)$} & \multirow[b]{2}{*}{ Total } & \multirow[b]{2}{*}{ OR $(95 \% \mathrm{Cl})$} & \multirow[b]{2}{*}{$p$-value } \\
\hline & $c l b+$ & $c l b-$ & & & \\
\hline Breastfeeding alone & 7 & 19 & 26 & $5.89(0.65$ to 53.11$)$ & 0.11 \\
\hline Natural delivery & 7 & 16 & 23 & & \\
\hline Cesarean section & 0 & 3 & 3 & & \\
\hline Mixed feeding with breastmilk and formula & 1 & 16 & 17 & 1 & \\
\hline Natural delivery & 1 & 11 & 12 & & \\
\hline Cesarean section & 0 & 5 & 5 & & \\
\hline
\end{tabular}

OR: odds ratio, $\mathrm{Cl}$ : confidence interval

such as providing counselling to expecting couples to help them become aware of the condition, testing the vaginal flora and altering the flora by external interferences [20] or providing couples assistance in choosing Cesarean section for delivering their infants. Of course, if future research establishes safe treatments to eradicate $c l b+E$. coli, such procedures could be performed before pregnancy or at a stable time during pregnancy to reduce the motherto-infant transmission of $c l b+E$. coli. To this end, we are currently analyzing the fecal samples from the mothers of the infants to fully understand the rates and modes of $c l b+E$. coli transmission from mothers and her infants.

Table 3 Screening of 58 food samples commonly consumed in Japan for clb + E. coli by PCR analysis

\begin{tabular}{|c|c|c|c|c|c|}
\hline Source & PCR & Source & PCR & Source & PCR \\
\hline \multicolumn{6}{|l|}{ Vegetable } \\
\hline bran pickles & - & broccoli & - & brown beech mushroom & - \\
\hline burdock root & - & cabbage & - & celery & - \\
\hline cherry tomato & - & Chinese cabbage & - & cloud ear mushroom & - \\
\hline cucumber & - & dried shiitake mushroom & - & enoki mushroom & - \\
\hline eryngii mushroom & - & ginger & - & Japanese ginger & - \\
\hline Japanese parsley & - & Japanese radish & - & kimchi & - \\
\hline Korean lettuce & - & lettuce & - & lotus root & - \\
\hline maitake mushroom & - & mesclun greens & - & olive oil & - \\
\hline onion & - & parsley & - & pea sprouts & - \\
\hline pickled Chinese cabbage & - & potherb mustard & - & red leaf lettuce & - \\
\hline salted plum & - & shiitake mushroom & - & soybean sprouts & - \\
\hline spinach & - & spring onion & - & tomato & - \\
\hline white radish sprouts & - & & & & \\
\hline \multicolumn{6}{|l|}{ Meat } \\
\hline beef & - & beef / ground meat & - & cattle / large intestine & - \\
\hline cattle / liver & - & cattle / small intestine & - & cattle / stomach & + \\
\hline chicken / ground meat & - & chicken / liver & - & pork / ground meat & - \\
\hline \multicolumn{6}{|l|}{ Seafood } \\
\hline clam & - & crab & - & freshwater clam & - \\
\hline salmon & - & shrimp & - & tuna & - \\
\hline \multicolumn{6}{|l|}{ Dairy products } \\
\hline butter & - & cheese & - & milk & - \\
\hline \multicolumn{6}{|l|}{ Others } \\
\hline honey & - & tap water & - & yogurt & - \\
\hline
\end{tabular}




\section{Methods}

\section{Participants and sample collection}

The subjects were 51 healthy infants from Tokyo metropolitan area in Japan. The size of the subject pool was deemed adequate for the study based on the known frequencies of $c l b+$ individuals found among healthy individuals (approximately $26.9 \%$ [14]) and child deliveries being carried out by Cesarean Sec. (24.4\% for 2005-2015 [21], and the rate is steadily increasing [22]) in Japan. If the frequency of $c l b+$ infants is similar to that of healthy adults, approximately three to four $c l b+$ infants are expected among 51 subjects $(51 \cdot 0.269 \cdot 0.244=3.3)$. Fecal samples were collected at birth to two to three days after birth (at the time of discharge within a few days after birth) and one month after birth (at the time of the one-month checkup). The collected feces were immediately placed in a sealed container and stored in a $-20{ }^{\circ} \mathrm{C}$ freezer until DNA extraction was performed.

\section{DNA extraction from fecal samples}

DNA was extracted from the frozen fecal samples with the bead beating method using a GNOME DNA Isolation Kit (MP Biomedicals). DNA quality was assessed with an Agilent 4200 TapeStation (Agilent Technologies). After the final DNA precipitation step, the DNA samples were resuspended in TE buffer and stored at $80{ }^{\circ} \mathrm{C}$ before the PCR analysis.

\section{DNA extraction from food materials}

Each food sample (10-50 g) obtained from grocery stores in Shizuoka, Japan, was added to $20 \mathrm{~mL}$ of EC medium (20 g peptone, $5 \mathrm{~g}$ lactose, $1.5 \mathrm{~g}$ bile salt, $4 \mathrm{~g}$ $\mathrm{K}_{2} \mathrm{HPO}_{4}, 1.5 \mathrm{~g} \mathrm{KH}_{2} \mathrm{PO}_{4}$ in $1 \mathrm{~L} \mathrm{H}_{2} \mathrm{O}$ ) in a sterilized bag. The mixture was incubated at $44.5^{\circ} \mathrm{C}$ for $24 \mathrm{~h}$. The mixture was filtered, and the filtrate was centrifugated at $10,000 \mathrm{~g}$ for $6 \mathrm{~min}$ at $4{ }^{\circ} \mathrm{C}$. The collected precipitate was suspended in $1 \mathrm{~mL}$ of sterile Milli-Q water. The suspension was plated on a MacConkey agar medium and incubated at $37{ }^{\circ} \mathrm{C}$ for $16 \mathrm{~h}$. The grown bacteria were cultured, and its genomic DNA was extracted by using a DNA isolation kit (QIAGEN). The isolated DNA was resuspended in TE buffer and stored at $-80^{\circ} \mathrm{C}$ before the PCR analysis.

\section{Confirmation of the presence of the $c l b$ gene cluster by PCR}

The extracted fecal DNA was subjected to PCR (SapphireAmp Fast PCR Master Mix, Takara) and qualitatively analyzed for $c l b B$ (a 9.6-kilobase gene encoding one of the colibactin biosynthetic enzymes) present in the DNA extract by amplifying the gene fragment using a primer set of clbB-F: 5'-TGTTCCGTTTTGTGTGGTTTCAGCG-3' and clbB-R: 5'-GTGCGCTGACCATTGAAGATTTCCG3 ' as described previously [11]. The correlation was analyzed by comparing the presence or absence of the $c l b B$ gene with the subject's birth method, diet content and sex.

\section{Statistical analysis}

Each experiment was performed at least three independent times. Representation of data as dot-plots and barand-whisker graphs is described in figure legends. The $t$ test for determining the statistically difference between the expected and observed frequencies was calculated using JMP (SAS Institute Inc.) and the NORM.DIST function in Microsoft Excel version 16.16.25.

\section{Supplementary Information}

The online version contains supplementary material available at https://doi. org/10.1186/s12866-021-02292-1.

\section{Additional file 1.}

\section{Acknowledgements}

The authors are grateful toward all the supports and would like to thank everyone who contributed to this study, including the parents and infants who provided the samples. We also thank all of the study site staff for cooperation on this project.

\section{Authors' contributions}

YT, MS, HM, YY, NM, KW1 and KW2 conceived and designed the study. JK, KH, NS and ES collected the fecal samples. YT and KW2 designed and performed PCR analysis. YI, MM1, HI, HS and MM2 performed the statistical analysis. All authors analyzed and discussed the results. KW1 and KW2 prepared the manuscript. All authors have read and approved the manuscript.

\section{Funding}

This study was funded by the Development of Innovative Research on Cancer Therapeutics from Japan Agency for Medical Research and Development (AMED) (K.Watanabe, 16ck0106243h0001; 19ck0106475h0001), Innovative Areas from MEXT, Japan (K.Watanabe, 16H06449), the Takeda Science Foundation (K.Watanabe), the Institution of Fermentation at Osaka (K.Watanabe), the Princess Takamatsu Cancer Research Fund (K.Watanabe, 16-24825), Kobayashi Foundation for Cancer Research (K.Watanabe), the Yakult Bio-Science

Foundation (K.Watanabe) and SECOM Science and Technology Foundation (K.Watanabe). The funding bodies had no role in the design of the study and collection, analysis, interpretation of data, or manuscript preparation.

Availability of data and materials

The datasets used and/or analyzed during the current study are available from the corresponding author on reasonable request.

\section{Declarations}

Ethics approval and consent to participate

A written letter of consent was prepared after obtaining informed consent from each of the parent to participate in this study. The study protocol was approved by the Ethical Committee of the National Institutes of Biomedical Innovation, Health and Nutrition, Japan (Approval No. 199-03).

Consent for publication

Not applicable.

\section{Competing interests}

The authors declare that they have no competing interests.

\section{Author details}

${ }^{1}$ Department of Pharmaceutical Sciences, University of Shizuoka, 422-8526 Shizuoka, Japan. ${ }^{2}$ Laboratory of Vaccine Materials, Center for Vaccine and Adjuvant Research, Laboratory of Gut Environmental System, Health and Nutrition (NIBIOHN), National Institutes of Biomedical Innovation, 567-0085 Ibaraki-city, Japan. ${ }^{3}$ Department of Pediatrics, Maternal and Child Health Center, Aiiku Clinic, 106-8580 Tokyo, Japan. ${ }^{4}$ Department of Human Nutrition, Tokyo Kasei Gakuin University, 194-0292 Tokyo, Japan. ${ }^{5}$ Department of Physical Activity Research, Health and Nutrition (NIBIOHN), National Institutes 
of Biomedical Innovation, 162-8636 Tokyo, Japan. ${ }^{6}$ School of Veterinary Medicine, Faculty of Veterinary Science, Nippon Veterinary and Life Science University, 180-8602 Tokyo, Japan. ${ }^{7}$ Department of Tumor Pathology, Hamamatsu University School of Medicine, 431- 3192 Shizuoka, Japan. ${ }^{8}$ Graduate School of Nutritional and Environmental Sciences, University of Shizuoka, 422-8526 Shizuoka, Japan. ${ }^{9}$ Department of Molecular-Targeting Cancer Prevention, Kyoto Prefectural University of Medicine, 602-8566 Kyoto, Japan.

Received: 4 November 2020 Accepted: 9 August 2021

Published online: 24 August 2021

\section{References}

1. Ferlay J, Colombet M, Soerjomataram I, Mathers C, Parkin DM, Piñeros M, et al. Estimating the global cancer incidence and mortality in 2018: GLOBOCAN sources and methods. Int J Cancer. 2019;144(8):1941-53.

2. Arnold M, Sierra MS, Laversanne M, Soerjomataram I, Jemal A, Bray F. Global patterns and trends in colorectal cancer incidence and mortality. Gut. 2017; 66(4):683-91.

3. Putze J, Hennequin C, Nougayrede JP, Zhang W, Homburg S, Karch H, et al. Genetic structure and distribution of the colibactin genomic island among members of the family Enterobacteriaceae. Infect Immun. 2009;77(11):4696-703.

4. Arthur JC, Perez-Chanona E, Muhlbauer M, Tomkovich S, Uronis JM, Fan TJ, et al. Intestinal inflammation targets cancer-inducing activity of the microbiota. Science. 2012;338(6103):120-3.

5. Healy AR, Nikolayevskiy H, Patel JR, Crawford JM, Herzon SB. A mechanistic model for colibactin-induced genotoxicity. J Am Chem Soc. 2016;138(48): 15563-70.

6. Kawanishi M, Hisatomi Y, Oda Y, Shimohara C, Tsunematsu Y, Sato M, et al, In vitro genotoxicity analyses of colibactin-producing E. coli isolated from a Japanese colorectal cancer patient. J Toxicol Sci. 2019;44(12):871-6.

7. Yoshikawa Y, Tsunematsu Y, Matsuzaki N, Hirayama Y, Higashiguchi F, Sato M, et al. Characterization of colibactin-producing Escherichia coli isolated from Japanese patients with colorectal cancer. Jpn J Infect Dis. 2020;73(6): 437-42.

8. Kawanishi M, Shimohara C, Oda Y, Hisatomi Y, Tsunematsu Y, Sato M, et al. Genotyping of a gene cluster for production of colibactin and in vitro genotoxicity analysis of Escherichia coli strains obtained from the Japan Collection of Microorganisms. Genes Environ. 2020;42:12.

9. Pleguezuelos-Manzano C, Puschhof J, Rosendahl Huber A, van Hoeck A, Wood HM, Nomburg J, et al. Mutational signature in colorectal cancer caused by genotoxic pks ${ }^{+}$E. coli. Nature. 2020;580(7802):269-73.

10. Dziubańska-Kusibab PJ, Berger H, Battistini F, Bouwman BAM, Iftekhar A, Katainen $\mathrm{R}$, et al. Colibactin DNA-damage signature indicates mutational impact in colorectal cancer. Nat Med. 2020;26(7):1063-9.

11. Hirayama $Y$, Tsunematsu Y, Yoshikawa $Y$, Tamafune R, Matsuzaki N, Iwashita $Y$, et al. Activity-based probe for screening of high-colibactin producers from clinical samples. Org Lett. 2019;21(12):4490-4.

12. Brotherton CA, Balskus EP. A prodrug resistance mechanism is involved in colibactin biosynthesis and cytotoxicity. J Am Chem Soc. 2013;135(9): 3359-62.

13. Bian X, Fu J, Plaza A, Herrmann J, Pistorius D, Stewart AF, et al. In vivo evidence for a prodrug activation mechanism during colibactin maturation. Chembiochem. 2013;14(10):1194-7.

14. Watanabe D, Murakami H, Ohno H, Tanisawa K, Konishi K, Tsunematsu Y, et al. Association between dietary intake and the prevalence of tumourigenic bacteria in the gut microbiota of middle-aged Japanese adults. Sci Rep. 2020;10:15221.

15. Arrieta MC, Stiemsma LT, Amenyogbe N, Brown EM, Finlay B. The intestinal microbiome in early life: health and disease. Front Immunol. 2014;5:427.

16. Payros D, Secher T, Boury M, Brehin C, Ménard S, Salvador-Cartier C, et al. Maternally acquired genotoxic Escherichia coli alters offspring's intestinal homeostasis. Gut Microbes. 2014;5(3):313-25.

17. Watt S, Lanotte P, Mereghetti L, Moulin-Schouleur M, Picard B, Quentin R. Escherichia coli strains from pregnant women and neonates: intraspecies genetic distribution and prevalence of virulence factors. J Clin Microbiol. 2003;41(5):1929-35.

18. Nowrouzian FL, Oswald E. Escherichia coli strains with the capacity for longterm persistence in the bowel microbiota carry the potentially genotoxic pks island. Microb Pathog. 2012;53(3-4):180-2.
19. Yassour M, Jason E, Hogstrom L, Arthur TD, Tripathi S, Siljander H, et al. Strain-level analysis of mother-to-child bacterial transmission during the first few months of life. Cell Host Microbe. 2018;24(1):146-54.e4.

20. Huang B, Fettweis JM, Brooks JP, Jefferson KK, Buck GA. The changing landscape of the vaginal microbiome. Clin Lab Med. 2014;34(4):747-61.

21. Shindo R, Aoki M, Yamamoto Y, Misumi T, Miyagi E, Aoki S. Optimal gestational weight gain for underweight pregnant women in Japan. Sci Rep. 2019;9(1):18129.

22. Yuda M. Public and social environment changes and caesarean section delivery choice in Japan. BMC Res Notes. 2018;11(1):633.

\section{Publisher's Note}

Springer Nature remains neutral with regard to jurisdictional claims in published maps and institutional affiliations.
Ready to submit your research? Choose BMC and benefit from:

- fast, convenient online submission

- thorough peer review by experienced researchers in your field

- rapid publication on acceptance

- support for research data, including large and complex data types

- gold Open Access which fosters wider collaboration and increased citations

- maximum visibility for your research: over $100 \mathrm{M}$ website views per year

At BMC, research is always in progress.

Learn more biomedcentral.com/submissions 